\title{
3-D EBSD Analysis of High Temperature Sintered YSZ; Evidence of Trace Cubic Phase
}

\author{
L.V. Saraf, ${ }^{*}$ S. Thevuthasan, ${ }^{*}$ B.W. Arey ${ }^{*}$ and L. R. Pederson ${ }^{* *}$ \\ * Environmental Molecular Sciences Laboratory, Pacific Northwest National Laboratory Richland \\ WA 99352 \\ ** Energy and Environmental Sciences Directorate, Pacific Northwest National Laboratory, \\ Richland WA 99352
}

We discuss our ongoing efforts related to the development of 3D-electron backscatter diffraction (EBSD) analysis capability for the materials used in solid oxide fuel cells (SOFC). A dual-beam focused ion beam (FIB)/scanning electron microscopy (SEM) capability from FEI (Helios) was configured with Oxford HKL EBSD system to analyze $8 \mathrm{~mol} \%\left(\mathrm{Y}_{2} \mathrm{O}_{3}\right)$ doped zirconia $\left(\mathrm{ZrO}_{2}\right)$ (YSZ). YSZ is one of the most important materials used as a SOFC electrolyte. Similarly $\mathrm{NiO}-\mathrm{YSZ}$ combination is one of the popular choices as an anode material in SOFC. The sample preparation for EBSD analysis was done by pressing YSZ powder (Tosoh Corp. Japan) uniaxially at $4500 \mathrm{~kg}$ and sintered at $1500{ }^{0} \mathrm{C} / 5 \mathrm{hrs}$ in air to get 1 " diameter and $3 \mathrm{~mm}$ thick dense pellet. YSZ pellet was further polished down to $\sim 1 \mu \mathrm{m}$ surface smoothness. After polishing, acetone and alcohol rinsed pellets were dried at $500{ }^{0} \mathrm{C} / 2 \mathrm{hrs}$. The YSZ pellet was prepared for 3D EBSD analysis in the dual-beam FIB/SEM system by successive $250 \mathrm{~nm}$ sectioning of $90^{\circ} \mathrm{YSZ}$ pellet wall by aligning it with incoming Ga ion beam tilted at $52^{\circ}$ w.r.t. electron beam. After each rough FIB sections, the sliced surfaces were fine cleaned by using low $\mathrm{kV}$ ion beam energy to further reduce surface Ga contribution and reduce ion beam induced possible amorphous layer thickness. The EBSD measurements were taken after $180^{\circ}$ rotation followed by alignment of the sectioned YSZ surface at $70^{\circ}$ with incoming electron beam. The process was repeated to get serial sections followed by data $3 \mathrm{D}$ reconstruction to get phase and crystallography information in the third dimension.

Even though FIB milling of the sample is a damaging process, by combining low energy YSZ sample milling with high-resolution EBSD data collection, one can obtain a true phase and crystallography of it in three dimensions. The sputtered YSZ depths for each slice could be controlled as low as 10-15 nm per slice. However due to large average grain size of YSZ in our case, $250 \mathrm{~nm}$ slicing was a preferred process. It is widely believed that $8 \mathrm{~mol} \%$ stabilized YSZ annealed at such high temperature is single phase with tetragonal structure. Our EBSD analysis clearly indicates evidence of cubic phase. Our data is in agreement with work by Badwal et.al.[1,2] which indicate that high temperature sintered YSZ undergoes a complex phase transformation where cubic phase transforms to metastable tetragonal; metastable tetragonal converts to cubic with high Y content and tetragonal phase with low Y content. The EBSD image in Figure 1 indicates YSZ EBSD pattern with a mixture of tetragonal and cubic phases. The EBSD map produced after 5 slices separated by $250 \mathrm{~nm}$ in YSZ is shown in Figure 2. In this figure, inverse pole figure grain orientation map along $\mathrm{x}$-direction in the case of YSZ is shown. As it is well known, pole figures in EBSD indicate crystallographic directions in which grains in a polycrystalline materials are distributed whereas inverse pole figure show how grains in a selected direction in the polycrystalline sample are distributed as oppose to reference frame of the crystal. Because YSZ is an important material used in SOFC, fundamental grain orientation properties are very important to understand the ionic transport behavior in YSZ. Thus, inverse pole figure maps are crucial to analyze YSZ transport properties. 


\section{References}

[1] S.P.S. Badwal, Solid State Ionics, 52 (1992) 23.

[2] S.P.S. Badwal, Journal of Materials Science, 22 (1987) 3231

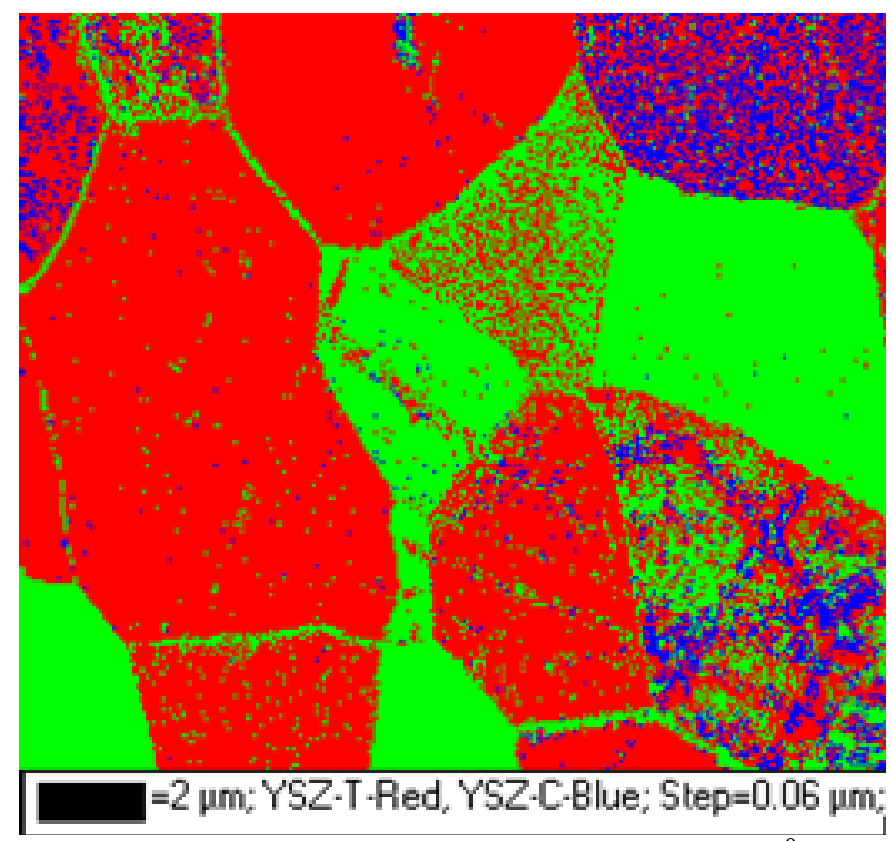

Figure 1. EBSD spectrum of YSZ surface annealed at $1500{ }^{0} \mathrm{C} / 5 \mathrm{hrs}$. Red color indicates dominating tetragonal phase whereas blue color indicates cubic YSZ as an impurity.

\section{EBSD (5 slices) - YSZ}

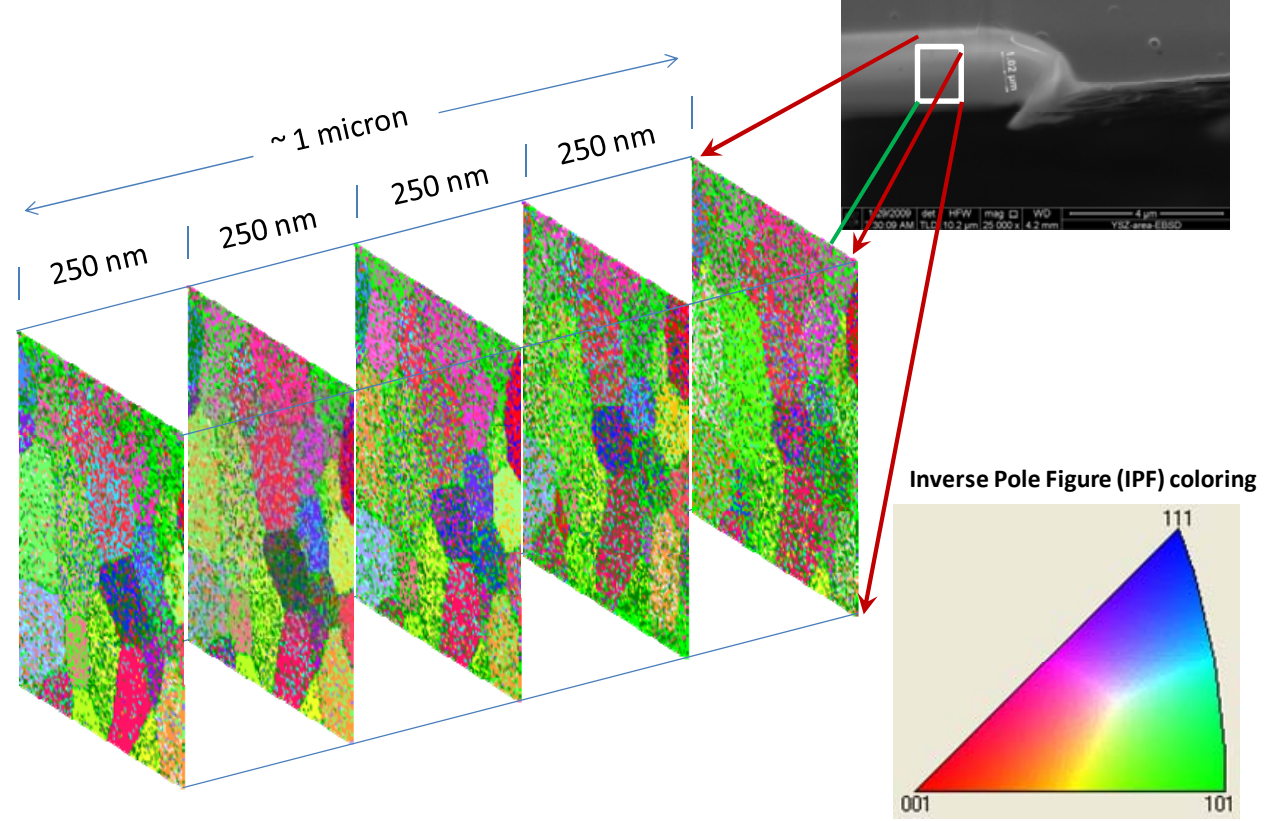

Figure 2. EBSD maps of 5 slices separated by $\sim 250 \mathrm{~nm}$ in $\mathrm{YSZ}$ annealed at $1500{ }^{\circ} \mathrm{C} / 5 \mathrm{hrs}$. 